\title{
Motion correction of whole-body PET data with a joint PET-MRI registration functional
}

\author{
Michael Fieseler ${ }^{1,2^{*}}$, Fabian Gigengack ${ }^{1,2 \dagger}$, Xiaoyi Jiang ${ }^{1,2 \dagger}$, Klaus P Schäfers ${ }^{2 \dagger}$ \\ From 35th Annual International Conference of the IEEE Engineering in Medicine and Biology Society: Workshop \\ on Current Challenging Image Analysis and Information Processing in Life Sciences \\ Osaka, Japan. 3-7 July 2013
}

\footnotetext{
* Correspondence: michael. fieseler@uni-muenster.de

${ }^{1}$ European Institute for Molecular Imaging, University of Münster, Mendelstrasse 11, 48149 Münster, Germany
}

\begin{abstract}
Respiratory motion is known to degrade image quality in PET imaging. The necessary acquisition time of several minutes per bed position will inevitably lead to a blurring effect due to organ motion. A lot of research has been done with regards to motion correction of PET data. As full-body PET-MRI became available recently, the anatomical data provided by MRI is a promising source of motion information. Current PET-MRIbased motion correction approaches, however, do not take into account the available information provided by PET data. PET data, though, may add valuable additional information to increase motion estimation robustness and precision.

In this work we propose a registration functional that is capable of performing motion detection in gated data of two modalities simultaneously. Evaluation is performed using phantom data. We demonstrate that performing a joint registration of both modalities does improve registration accuracy and PET image quality.
\end{abstract}

\section{Introduction}

Respiratory motion is known to impair image quality as well as quantification in positron emission tomography (PET) [1]. As the acquisition of PET takes several minutes per bed position, organ motion due to respiration cannot be avoided and will thus result in blurred images. By using gating methods, the acquired PET data can be divided into different motion phases. Gating reduces the amount of motion contained within each gate to a large extent, yet at the expense of reduced statistics and thereby image quality [2,3]. To alleviate this, motion between gates can be estimated and subsequently be used to correct PET data for motion, resulting in a single image volume with reduced motion artifacts and full statistics. Various approaches for motion estimation of gated PET data have been studied, including optical flow [4], B-spline based methods [5], and registration methods including mass-preservation [6].

The use of 4D-CT data for motion correction has been proposed [7]. The advantage of this approach lies in the usage of anatomical data, which is independent of tracer uptake. Acquisition of 4D-CT data, however, increases the radiation burden for the patient.

Whole body PET-MRI is promising regarding PET motion correction. High resolution MR data may allow for a precise motion estimation independent of tracer uptake

(c) 2014 Fieseler et al.: licensee BioMed Central Ltd. This is an Open Access article distributed under the terms of the Creative Commons Attribution License (http://creativecommons.org/licenses/by/2.0), which permits unrestricted use, distribution, and reproduction in any medium, provided the original work is properly cited. The Creative Commons Public Domain Dedication waiver (http:// creativecommons.org/publicdomain/zero/1.0/) applies to the data made available in this article, unless otherwise stated. 
and without additional radiation burden for the patient. The feasibility of PET-MRIbased motion correction of PET data has been demonstrated already, e.g., using hardware phantoms [8], animals [9], and simulation data [10]. A commonality of current approaches to MR-based motion correction is that a 4D MR dataset is acquired from which motion is estimated and subsequently used in the correction of PET data. Strategies for the generation of 4D MR include acquisition of 2D slices with subsequent reordering [11], fast, consecutive acquisitions of 3D volumes [12], and sorting of k-space data during or after acquisition [13,14]. Fayad et al. propose an approach where motion and image data are estimated simultaneously from the acquired MR data [15].

The motion information contained in PET data, however, remains unused in these approaches. In clinical routine, time for the acquisition of MR data needed for motion correction may be limited, as clinical protocols may demand for further, diagnostic sequences [16]. Since time is proportional to image quality in MR, limited acquisition time may not allow to exploit the full potential of MR, resulting in poorer image quality than technically possible. Accordingly, all available information for motion estimation, including PET data, should be used.

Further, both modalities may contribute valuable information to the motion detection process. In MR, e.g., the lungs give relatively little signal due to their low proton density [14]. Integrating information from PET data, if, e.g., active lesions are present in the lungs, may benefit motion estimation. Using information from both modalities should result in more reliable registration results. In the present work we propose an approach that uses information from both modalities by combining two registration functionals into a joint functional.

\section{Methods}

For motion correction approaches in PET-MRI, proper synchronisation of MR and PET is mandatory. The motion determined from MR has to be related to the PET data with respect to time. For the approach described in the following, we assume a gated PET dataset as well as a series of MR datasets. We assume that each PET gate corresponds to one MR gate with respect to its motion phase. The feasibility of creating corresponding phases has been demonstrated, e.g., in [14].

In the following we describe the proposed registration functional, followed by a description of the phantom data used in this work.

\section{Registration functional}

Registration can be formulated as the problem of finding the transformation $y$ that minimizes

$$
\mathcal{J}(\gamma)=\mathcal{D}(\mathcal{T}(\gamma), \mathcal{R})+\alpha \cdot \mathcal{S}(\gamma)
$$

where $\mathcal{D}$ is a distance functional, $\mathcal{R}$ is the reference volume, $\mathcal{T}$ is the template volume to be registered, and $\mathcal{S}$ is a regularizer penalizing unfavourable transformations. The scalar value $\alpha$ weights the influence of the regularizer. For non-rigid transformations, $y$ is chosen as a non-parametric transformation (one vector per voxel).

The registration functional $\mathcal{J}(y)$ in Equation (1) could be applied to each modality independently. Ideally, the resulting transformations for both modalities, PET and MR, 
should be equal. This, however, will not occur in reality, as both modalities do not provide the same, but complimentary information. The objective is thus to combine two registration functionals into one:

$$
\mathcal{J}(y)=\mathcal{D}\left(\mathcal{T}_{\mathrm{MR}}(y), \mathcal{R}_{\mathrm{MR}}\right)+\beta \cdot \mathcal{D}\left(\mathcal{T}_{\mathrm{PET}}(y), \mathcal{R}_{\mathrm{PET}}\right)+\alpha \cdot \mathcal{S}(\gamma)
$$

Here, $\mathcal{R}_{\mathrm{MR}}$ and $\mathcal{R}_{\text {PET }}$ denote two reference volumes and $\mathcal{T}_{\mathrm{MR}}$ and $\mathcal{T}_{\mathrm{PET}}$ the template volumes. The scalar value $\beta$ allows to weight the influence of the data term for PET.

In the registration functional in Equation (2), the deformation is represented by a common grid $y$ for both modalities. Since the input data will not necessarily share the same resolution, resampling to a common grid is performed. Here, we chose a common grid of $2 \mathrm{~mm}^{3}$ voxel size.

For $\mathcal{S}$ we use a hyperelastic regularizer for its ability to penalize changes in volume, area, and length separately. The hyperelastic regularizer $\mathcal{S}^{\text {hyper is defined as }}$

$$
\mathcal{S}^{\text {hyper }}(\gamma)=\alpha_{l} \cdot \mathcal{S}^{\text {length }}\left(\gamma-\gamma^{\text {ref }}\right)+\alpha_{a} \cdot \mathcal{S}^{\text {area }}(\gamma)+\alpha_{v} \cdot \mathcal{S}^{\text {volume }}(y),
$$

where $y^{\text {ref }}$ is a reference grid, given by the identity transformation in our case. The three summands control changes in length, surface area, and volume. Parameters for the hyperelastic regularizer were chosen empirically as $\alpha_{l}=1, \alpha_{a}=0.1, \alpha_{v}=1$. Throughout all experiments in this paper, we keep $\alpha_{l}, \alpha_{a}, \alpha_{v}$ fixed and vary the regularization strength by changing $\alpha$ in Eq. (2). For further details regarding the regularizer we refer to [17].

As the distance functional $\mathcal{D}$, we choose the sum of squared differences (SSD) for both PET and MR. The functional was implemented using the Matlab-based FAIR toolbox [18]. For all registration experiments, linear interpolation was used and a multi-level approach using a downscaling factor of 0.5 was applied. Optimiziation was performed using a Gauss-Newton scheme with a preconditioned conjugate gradient solver.

\section{Phantom data}

In the present work we use data generated using a software phantom for evaluation. This allows us to compare motion estimates against ground-truth motion data. The XCAT phantom [19] is widely used as the basis for the simulation of imaging modalities and the evaluation of correction methods, e.g., in [6,20,21]. Using the XCAT phantom, we created an artificial PET-MRI dataset as described in the following.

The XCAT phantom was set to a maximum diaphragm motion of $2 \mathrm{~cm}$. We selected eight frames representing the full range from inspiration to expiration [22].

For the creation of MR data a labelled XCAT dataset of $1 \mathrm{~mm}^{3}$ resolution was created. This dataset and known tissue values for $T_{1}, T_{2}$, and proton density [23,24] were used as an input to the freely available MR simulation software SIMRI [25]. We simulated an MR acquisition of stacked 2D slices covering the thorax. For respiratory motion, the largest amount of motion can be expected in the cranio-caudal and anterior-posterior directions. Thus, a sagittal slice orientation was chosen to capture these directions in-plane. For the phantom dataset used here, the maximum extents of motion are $2.5 \mathrm{~mm}$ (left-right), 10.9 $\mathrm{mm}$ (anterior-posterior), and $25.2 \mathrm{~mm}$ (head-feet). The following MR parameters were used: gradient echo, TE/TR $10 \mathrm{~ms} / 30 \mathrm{~ms}, 12^{\circ}$ flip angle, $256 \times 256$ pixels, $2 \mathrm{~mm}$ pixel size in-plane, slice spacing $1 \mathrm{~cm}$, slice thickness $1 \mathrm{~cm}$. 
For PET data creation an XCAT dataset with lesions added to the lungs and the liver was created (heart $50 \mathrm{kBq} / \mathrm{ml}$, liver $20 \mathrm{kBq} / \mathrm{ml}$, background $2 \mathrm{kBq} / \mathrm{ml}$, lesions $50 \mathrm{kBq} / \mathrm{ml}$, lesion diameter $5 \mathrm{~mm}$ ). Sinograms were created by forward-projection using the geometry of the Siemens Biograph mMR scanner. Poisson noise was added to the sinograms. Additionally, attenuation was added using the attenuation maps provided by the XCAT phantom. For comparison, a second phantom as described above was created, but without lesions. Subsequently, the sinograms were reconstructed. All projections and reconstructions were performed using EMRECON [26]. The generated data is shown in Figure 1.

\section{Evaluation}

Using the phantom data described above allows for evaluation based on ground-truth motion as well as ground-truth activity data. First, we set $\beta=0$, thereby performing an MR-based registration and use varying values of $\alpha$ to determine the best result for an MR-based registration.

Using the determined registration parameters, we compute registrations for increasing values of $\beta$, thus adding increasing amounts of PET information to the registration.

The resulting deformation fields $y$ are compared to the ground-truth motion provided by the XCAT phantom by means of the averaged endpoint error (AEE) defined by

$$
\operatorname{AEE}\left(y, y^{\mathrm{GT}}\right)=\frac{1}{|\Omega|} \cdot \sum_{x \in \Omega} \sqrt{\sum_{i=1}^{3}\left(y_{i}(x)-y_{i}^{\mathrm{GT}}(x)\right)^{2}}
$$

where $\Omega$ is the image domain, $y_{i}$ the $\mathrm{i}$-th component of vector $y$, and $y^{\mathrm{GT}}$ the ground-truth vector. Here, we examine averaged values of the AEE for all gates.

Further, the computed motion estimates are used to perform a motion correction of the dataset. The PET gates are warped using the computed motion and averaged. We evaluate correlation values of the corrected images as well as the recovered activities in the three lesion regions. For all registrations, attenuated PET data are used. For evaluation of correlation coefficients and activity recovery, attenuation corrected PET data are used.

\section{Results}

\section{Registration accuracy}

Figure 2 shows the average endpoint error for registrations with increasing regularization strength $\alpha$. A value of $\alpha=75$ minimizes the overall average endpoint error.

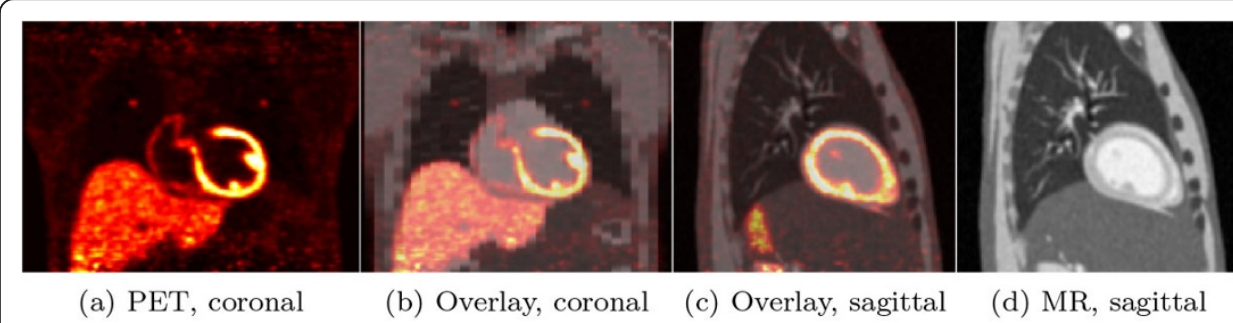

Figure 1 Phantom Data (a) Simulated PET data, coronal view. Visible are lesions in both lungs and in the liver. (b) Overlay of PET and MR, coronal view. (c) Overlay of PET and MR, sagittal view. (d) Simulated MR data, sagittal slice. 


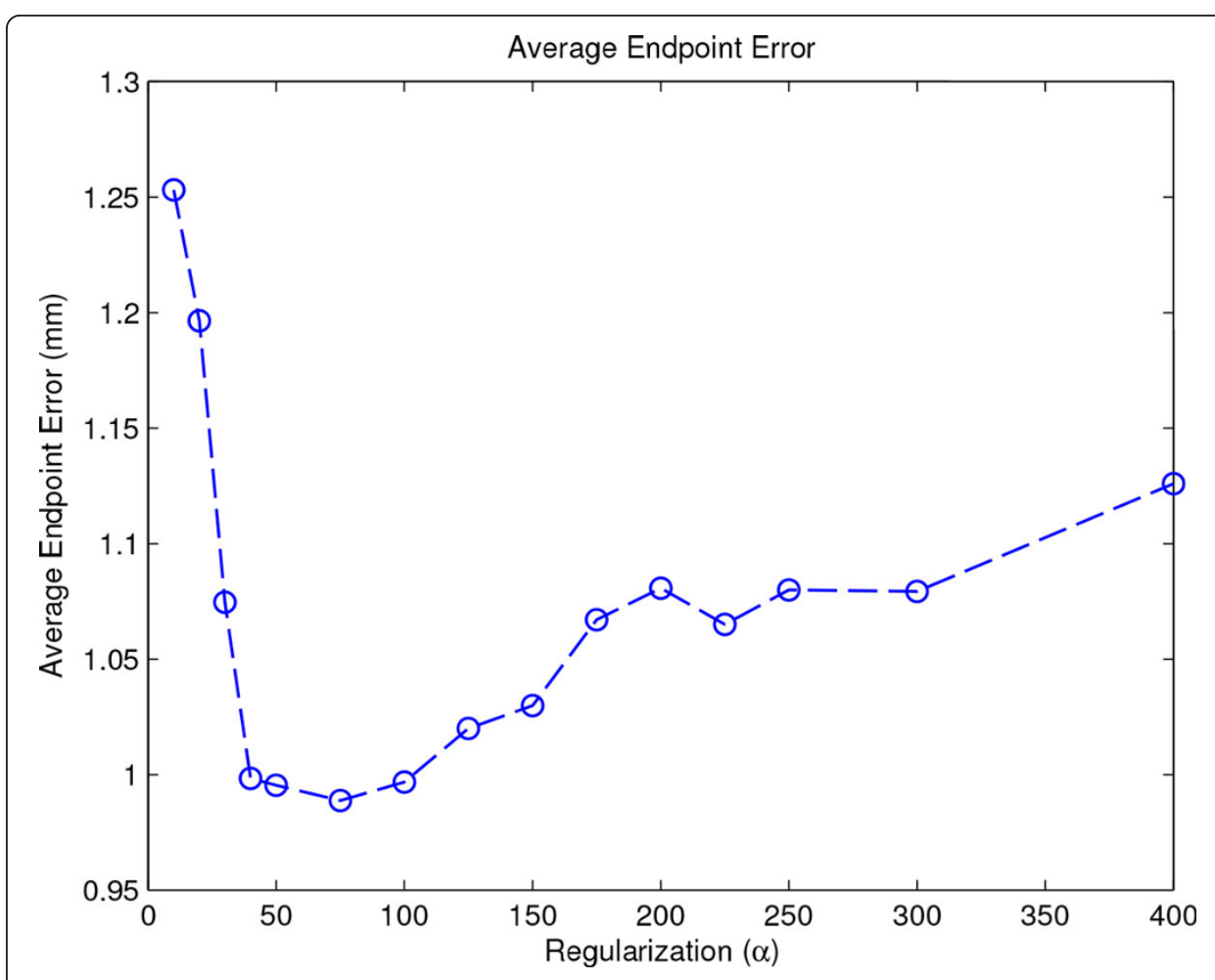

Figure 2 Average Endpoint Error for MR-only registration. The figure shows the global average endpoint error (AEE) for a range of regularization values $\alpha$. PET weight $\beta$ is kept to zero here. A minimum at $\alpha=75$ is observable.

Figure 3 shows results for increasing values of $\beta$. By increasing $\beta$, AEEs for the heart and the lung are slightly reduced, whereas the global error and the error in the liver region increases slightly.

In Figure $4 \mathrm{AEEs}$ for the lesion regions are shown. In Figure 4a we show results for the phantom with added lesions as described above. For all three lesion regions a significant decrease in AEE can be observed. To examine if the decrease in AEE is indeed caused by a better registration due to the information provided by the lesions, we

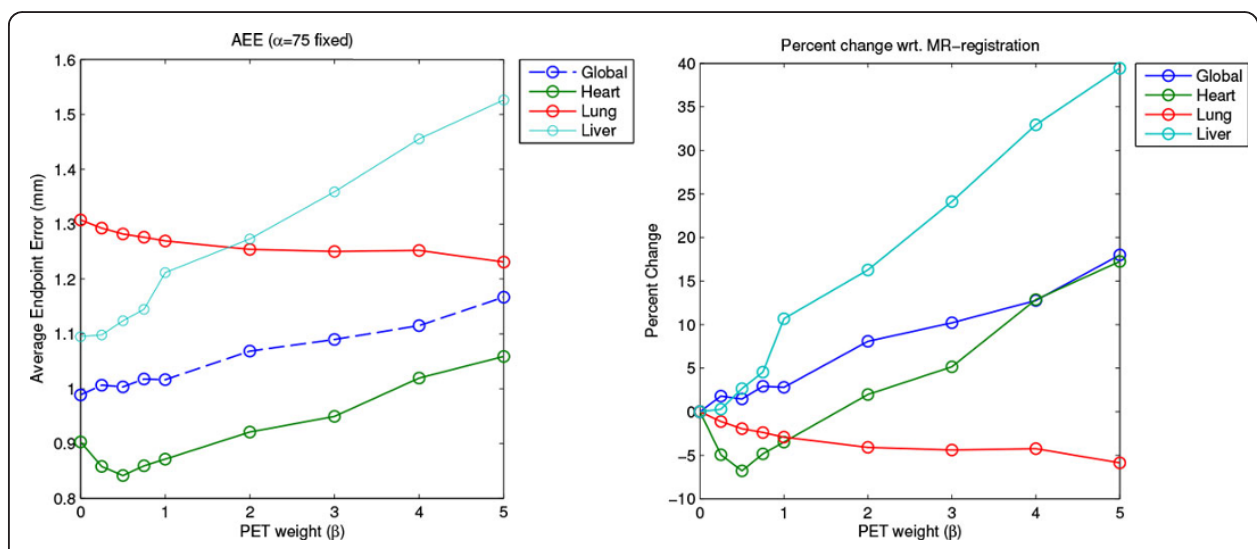

Figure 3 Average Endpoint Error for varying degrees of PET influence. Shown is the AEE for local regions and globally for increasing additions of PET to the registration (weight $\beta$ ). The upper plot gives the AEE, the lower plot the percentage change with respect to the MR-only registration $(\alpha=75, \beta=0)$. 


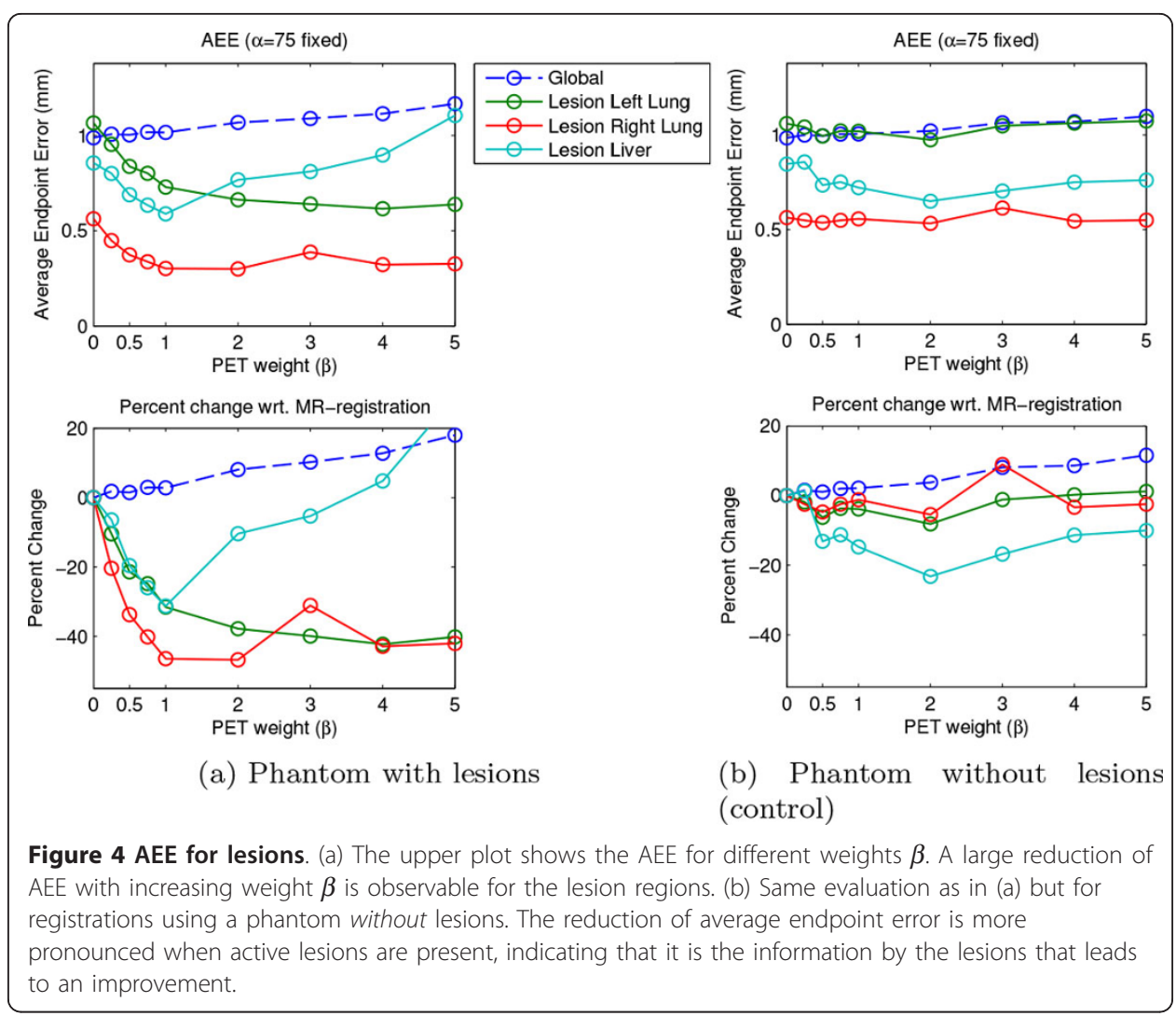

repeated this experiment with the same phantom but without lesions. Results for this experiment are shown in Figure 4b. Apart from the liver lesion region, the AEE for the lesions remains almost unchanged for increasing values of $\beta$.

\section{Correlation coefficients}

After applying the motion estimates to the input data, we evaluated the average correlation of all corrected PET gates with respect to the target gate. To evaluate if adding PET information to the registration impairs correlation values for MR data, the motion estimates were used to warp the MR data as well. Results are given in Figure 5, for full image data (global) as well as for the heart region. The correlation of the PET data is increased with increasing values of $\beta$ while correlation values of the MR data decrease only slightly.

\section{Recovered activity in lesions}

In Figure 6, evaluation results regarding recovered activity in the three lesions are given as average and maximum activity. Adding information from PET data leads to a better recovery of activity. With very large values of $\beta$, activity recovery is decreasing again.

\section{Discussion}

Using the joint registration approach, local improvements are observable. For the heart, the lungs, and the lesions (lungs and liver), improvements in terms of registration error (AEE) are achieved. In particular the lesions in the lungs show a large reduction in registration error when PET data are added to the registration. For the liver region, an 


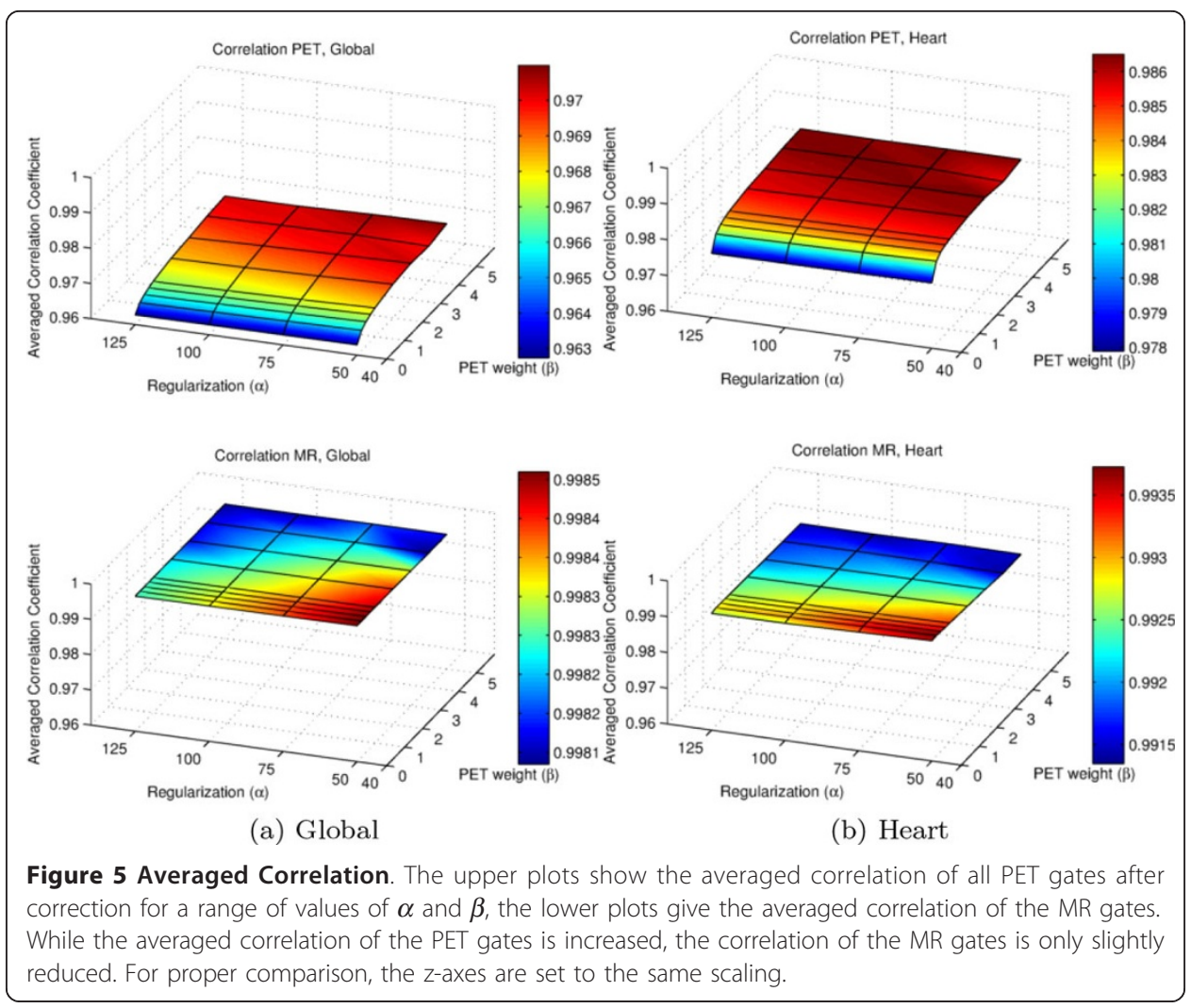

increase of the AEE is observable. For the area of the lesion added to the liver, though, the AEE is decreased. With the exception of the liver region in toto, those regions that exhibit tracer uptake in PET seem to contribute to a better motion estimation result. Correlation of PET data is improved if PET data is added to the registration, indicating a benefit. Additionally, the lower registration error leads to a slightly better recovery of the activity in the lesions. Globally, a slight increase of the AEE is observable with increasing values of $\beta$.

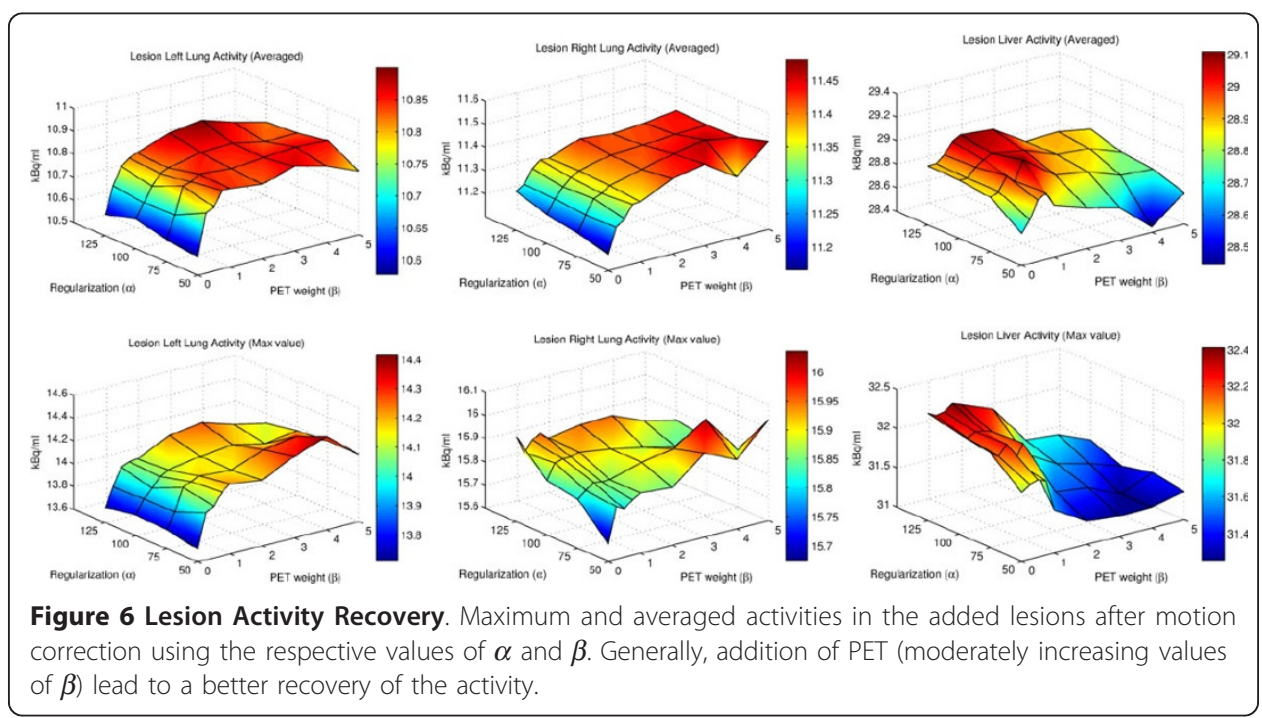


The results presented here indicate that a benefit of using motion information from both modalities, MR and PET, is achievable. Certainly, the extent of the benefit will depend on many factors, with one major factor being the image quality of the two image modalities. The amount of remaining motion-induced blurring within the individual MR and PET gates will certainly limit the precision to which motion can be estimated. The extent to which PET can contribute will as well depend on the image quality, determined by factors like, e.g., type of injected tracer, injected dose, tracer uptake, and acquisition time. The phantom data used here does not contain artifacts other than noise. Particularly, we did not simulate motion artifacts which are likely to occur during MR acquisitions.

\section{Conclusion}

We have presented a joint registration functional that makes use of motion information derived from PET and MR data simultaneously. In this approach, motion information from both modalities is used. We demonstrated that the proposed method leads to a lower local registration error and better recovery of lesion activity, thus using information from both modalities simultaneously is beneficial regarding motion correction. As a result, clinical scenarios involving lesion quantification might in particular benefit from the proposed method.

In future work, we will evaluate our approach for a broader range of data, including cardiac motion. This will include the addition of mass-preservation [6]. Certainly, the phantom data used in this work does only approximate reality. Thus, we will evaluate the proposed approach on real phantom data.

Competing interests

The authors declare that they have no competing interests.

\section{Acknowledgements}

This work was partly funded by the German Research Foundation (Deutsche Forschungsgemeinschaft), Sonderforschungsbereich SFB $656 \mathrm{MoBil}$ (project B3), and a research grant from Siemens Healthcare, Erlangen, Germany. We acknowledge support by Deutsche Forschungsgemeinschaft and Open Access Publication Fund of University of Muenster.

This article has been published as part of BioMedical Engineering OnLine Volume 13 Supplement 1, 2014: Selected articles from the 35th Annual International Conference of the IEEE Engineering in Medicine and Biology Society: Workshop on Current Challenging Image Analysis and Information Processing in Life Sciences. The full contents of the supplement are available online at http://www.biomedical-engineering-online.com/supplements/13/S1

\section{Authors' details}

${ }^{1}$ European Institute for Molecular Imaging, University of Münster, Mendelstrasse 11, 48149 Münster, Germany.

2Department of Computer Science, University of Münster, Einsteinstrasse 62, 48149 Münster, Germany.

Published: 28 February 2014

\section{References}

1. Nehmeh S, Erdi Y, Ling C, Rosenzweig K, Squire O, Braban L, Ford E, Sidhu K, Mageras G, Larson S, et al: Effect of respiratory gating on reducing lung motion artifacts in pet imaging of lung cancer. Medical physics 2002, 29:366.

2. Büther F, Ernst I, Dawood M, Kraxner P, Schäfers M, Schober O, Schäfers KP: Detection of respiratory tumour motion using intrinsic list mode-driven gating in positron emission tomography. European Journal of Nuclear Medicine and Molecular Imaging 2010, 37:2315-2327.

3. Dawood M, Buether F, Lang N, Schober O, Schaefers KP: Respiratory gating in positron emission tomography: A quantitative comparison of different gating schemes. Medical Physics 2007, 34(7):3067.

4. Dawood M, Büther F, Jiang X, Schäfers KP: Respiratory motion correction in 3-D PET data with advanced optical flow algorithms. IEEE Transactions on Medical Imaging 2008, 27(8):1164-1175.

5. Bai W, Brady M: Regularized b-spline deformable registration for respiratory motion correction in PET images. Physics in Medicine and Biology 2009, 54(9):2719.

6. Gigengack F, Ruthotto L, Burger M, Wolters CH, Jiang X, Schäfers KP: Motion correction in dual gated cardiac PET using mass-preserving image registration. IEEE Transactions on Medical Imaging 2012, 31(3):698-712. 
7. Lamare F, Carbayo MJL, Cresson T, Kontaxakis G, Santos A, Rest CCL, Reader AJ, Visvikis D: List-mode-based reconstruction for respiratory motion correction in PET using non-rigid body transformations. Physics in Medicine and Biology 2007, 52(17):5187.

8. Tsoumpas C, Mackewn J, Halsted P, King A, Buerger C, Totman J, Schaeffter T, Marsden P: Simultaneous PET-MR acquisition and MR-derived motion fields for correction of non-rigid motion in PET. Annals of Nuclear Medicine 2010, 24(10):745-750.

9. Chun SY, Reese TG, Ouyang J, Guerin B, Catana C, Zhu X, Alpert NM, El Fakhri G: MRI-based nonrigid motion correction in simultaneous PET/MRI. Journal of Nuclear Medicine 2012, 53(8):1284-1291.

10. Dikaios N, Izquierdo-Garcia D, Graves M, Mani V, Fayad Z, Fryer T: MRI-based motion correction of thoracic PET: initial comparison of acquisition protocols and correction strategies suitable for simultaneous PET/MRI systems. European Radiology 2012, 22:439-446.

11. Würslin C, Schmidt H, Martirosian P, Boss A, Schwenzer NF, Stegger L: Respiratory motion correction in oncologic PET using t1-weighted MR imaging on a simultaneous whole-body PET/MR system. Journal of Nuclear Medicine 2013, 54(3):464-471.

12. Tsoumpas C, Buerger C, King AP, Mollet P, Keereman V, Vandenberghe S, Schulz V, Schleyer P, Schaeffter T, Marsden PK: Fast generation of 4D PET-MR data from real dynamic MR acquisitions. Physics in Medicine and Biology 2011, 56(20):6597.

13. Ouyang J, Li Q, Fakhri GE: Magnetic resonance-based motion correction for positron emission tomography imaging Seminars in Nuclear Medicine 2013, 43(1):60-67.

14. Dutta J, El Fakhri G, Huang C, Petibon Y, Reese TG, Li Q: Respiratory motion compensation in simultaneous PET/MR using a maximum a posteriori approach. Biomedical Imaging (ISBI), 2013 IEEE 10th International Symposium On IEEE; 2013, 800-803.

15. Fayad H, Odille F, Felblinger J, Visvikis D: A generic PET/MRI respiratory motion correction using a generalized reconstruction by inversion of coupled systems (GRICS) approach. Nuclear Science Symposium and Medical Imaging Conference (NSS/MIC), 2012 IEEE IEEE; 2012, 2813-2816.

16. Bailey DL, Beyer T, Boellaard R, Gückel B, Hellwig D, Herzog H, Pichler BJ, Quick HH, Sabri O, et al: Summary report of the first international workshop on PET/MR imaging, march 19-23, 2012, tübingen, germany. Molecular Imaging and Biology 2013, 1-11.

17. Burger $M$, Modersitzki J, Ruthotto L: A hyperelastic regularization energy for image registration. SIAM Journal on Scientific Computing 2012

18. Modersitzki J: Fair: Flexible Algorithms for Image Registration (Fundamentals of Algorithms). Society for Industrial and Applied Mathematics, Philadelphia 2009.

19. Segars WP, Sturgeon G, Mendonca S, Grimes J, Tsui BM: $4 D$ XCAT phantom for multimodality imaging research. Medical physics 2010, 37(9):4902-4915.

20. Xia T, Alessio AM, De Man B, Manjeshwar R, Asma E, Kinahan PE: Ultra-low dose CT attenuation correction for PET/CT. Physics in Medicine and Biology 2012, 57(2):309.

21. Tabary J, Marache-Francisco S, Valette S, Segars WP, Lartizien C: Realistic X-ray CT simulation of the XCAT phantom with SINDBAD. Nuclear Science Symposium Conference Record (NSS/MIC), 2009 IEEE 2009, 3980-3983.

22. Dawood M, Büther F, Stegger L, Jiang X, Schober O, Schäfers M, Schäfers KP: Optimal number of respiratory gates in positron emission tomography: A cardiac patient study. Medical physics 2009, 36:1775.

23. Stanisz GJ, Odrobina EE, Pun J, Escaravage M, Graham SJ, Bronskill MJ, Henkelman RM: T1, T2 relaxation and magnetization transfer in tissue at 3T. Magnetic Resonance in Medicine 2005, 54(3):507-512.

24. Tobon-Gomez C, Sukno F, Bijnens BH, Huguet M, Frangi A: Realistic simulation of local image appearance of cardiac magnetic resonance imaging using a virtual phantom population. CI2BM09 - MICCAI Workshop on Cardiovascular Interventional Imaging and Biophysical Modelling, London, Royaume-Uni 2009, 132-140.

25. Benoit-Cattin H, Collewet G, Belaroussi B, Saint-Jalmes H, Odet C: The SIMRI project: a versatile and interactive MRI simulator. Journal of Magnetic Resonance 2005, 173(1):97-115.

26. Kösters T, Schäfers KP, Wübbeling F: EMrecon: An expectation maximization based image reconstruction framework for emission tomography data. Nuclear Science Symposium and Medical Imaging Conference (NSS/MIC), 2011 IEEE 2011, 4365-4368.

doi:10.1186/1475-925X-13-S1-S2

Cite this article as: Fieseler et al:: Motion correction of whole-body PET data with a joint PET-MRI registration functional. BioMedical Engineering OnLine 2014 13(Suppl 1):S2

\section{Submit your next manuscript to BioMed Central and take full advantage of:}

- Convenient online submission

- Thorough peer review

- No space constraints or color figure charges

- Immediate publication on acceptance

- Inclusion in PubMed, CAS, Scopus and Google Scholar

- Research which is freely available for redistribution 